\title{
Inhibition and disinhibition of an operant response as a function of the amount and type of prior training*†
}

\author{
C. J. BRIMER \\ Dalhousie University, Halifax, N.S., Canada
}

A total of 132 rats were employed in two experiments designed to investigate the effect of an extraneous stimulus (light) presented at different stages of extinction. The animals were initially trained with either a CRF or VI food-reinforcement schedule. Presenting the light early in extinction led to inhibition in the CRF animals and disinhibition in the VI Ss. Later in extinction both groups showed the disinhibition effect. The results suggested that the low response rate necessary to demonstrate disinhibition was a function of both the amount and type of original reinforced training.

Previous studies (Brimer, 1970) have demonstrated that the operant leverpress situation can provide a sensitive tool with which to investigate the Pavlovian phenomena of inhibition and disinhibition. The general finding has been that when the response is reinforced and, consequently, the response rate is high, the introduction of an extraneous stimulus produces a response decrement. In Pavlovian terminology the stimulus produces external inhibition. When, on the other hand, the response rate is suppressed by some inhibitory operation, such as experimental extinction, the introduction of the same extraneous stimulus results in a response increment. This latter effect, of course, represents the disinhibition phenomenon. The results of previous studies (Brimer, 1970) suggest that disinhibition will occur only when the response rate lies within some critical low range of values. The present experiments were designed to investigate how disinhibition might vary as a function of the amount and type of prior reinforced training. In Experiment 1, animals were first given different amounts of acquisition training under either a continuous or partial reinforcement schedule, followed by extinction training and disinhibition testing.

\section{EXPERIMENT 1 \\ Method}

The Ss were 72 male hooded rats, maintained at approximately $75 \%$ of their ad lib weights throughout the experiment. There were 12 Ss assigned to each of six experimental groups. The apparatus consisted of three standard Grason-Stadler operant-conditioning units, controlled by relays, timers, and counters located in an

*This research was supported by Research Grant APA-105 to the author from the National Research Council of Canada.

t The author wishes to express his appreciation to David Boyle and Robert Sainsbury for experimental assistance. adjacent room. Following initial leverpress training, half the animals were trained with a continuous (CRF), and the other half with a 1-min variable-interval (VI), food-reinforcement schedule. For all animals a daily acquisition-training session consisted of 50 reinforced responses. The three groups of Ss that were run under each training schedule received 100,300 , or 500 reinforcements prior to extinction training.

When an animal, during extinction, went 3 min without a response, the extraneous stimulus was presented. The extraneous stimulus was a $3-\mathrm{min}$ light (all training was done in complete darkness) of approximately $2 \cdot \mathrm{fc}$ intensity. Each animal received one stimulus and one dummy trial, the order of presentation being counterbalanced between Ss. A dummy trial simply consisted of counting the number of responses that occurred in the 3-min interval following completion of the $3-\mathrm{min}$ no-response criterion. Thus, disinhibition could be calculated by comparing an animal's response rate during the 3-min stimulus with its rate during the comparable 3-min dummy trial.

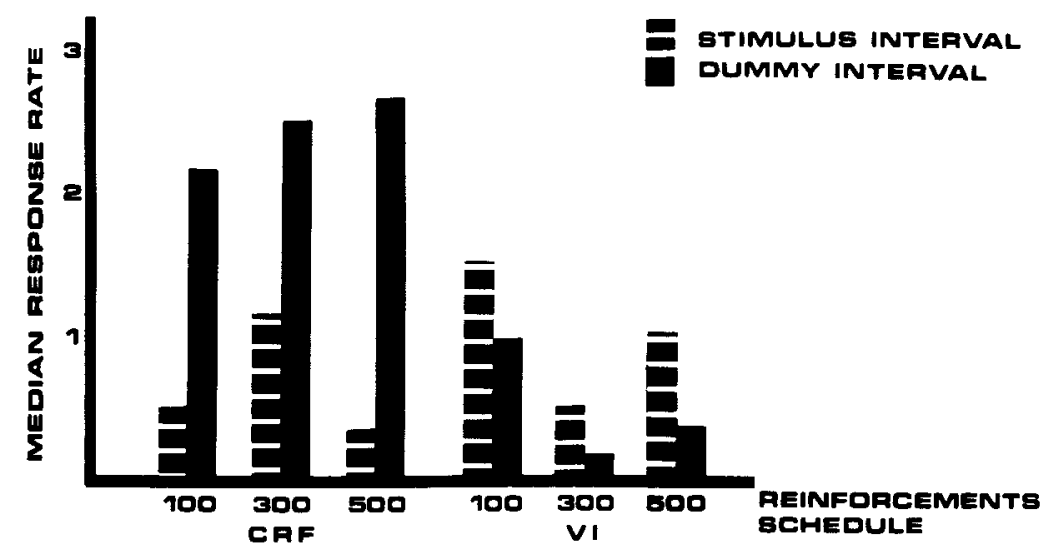

Fig. 1. Median rate (responses per minute) during stimulus and dummy intervals as a function of training conditions. 500 reinforcements $(T=4.5, p<.02)$.

Looking at the CRF animals, it is found, somewhat surprisingly, that a significant proportion of the Ss $(24.5 / 36, p<.05)$ responded less during the stimulus than during dummy intervals. Indexing this inhibition by difference scores revealed no differences among the three CRF groups $(\mathrm{H}=0.5, \mathrm{p}>.05)$. However, with individual tests, only the 500 reinforcement animals could be shown to make significantly fewer stimulus than dummy responses $(T=5.0, p<.05)$.

\section{Discussion}

In Experiment 1 it was found that animals originally trained under a partial-reinforcement schedule would accelerate responding when presented with an extraneous stimulus during extinction. Furthermore, there was some suggestion that this disinhibition effect might be more pronounced for those Ss that received the greatest amount of acquisition training. In contrast, animals who were trained under a continuous-reinforcement schedule suppressed responding when the same stimulus was presented during extinction. Again, there was some tendency for this

The results of the disinhibition test are presented in Fig. 1. Focusing first on the VI-trained animals reveals that a significant proportion of the Ss $(25 / 36, p<.05)$ responded more during the stimulus than during the dummy interval. In order to index the magnitude of this disinhibition effect, each $S$ was assigned a difference score, calculated by subtracting the number of dummy responses from the number of stimulus responses. In terms of this measure there were no differences among the three VI groups $(\mathrm{H}=1.3$, $p>.05$ ). However, when the three groups were tested individually for disinhibition (by comparing the number of stimulus and dummy responses), a significant effect was found only for the animals that received

.

.



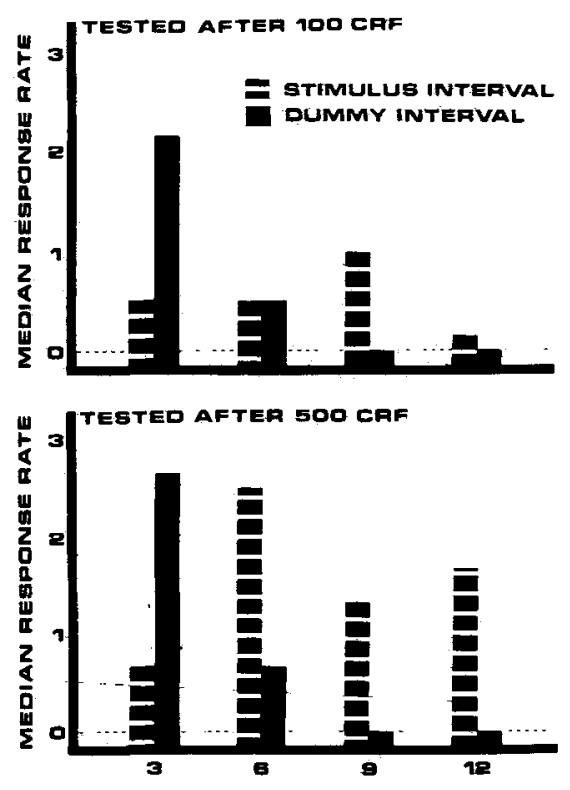

EXTINCTION CRITERION (IN MIN.)

Fig. 2. Median rate (responses per minute) during stimulus and dummy intervals as a function of the amount of acquisition and extinction training.

inhibition effect to be greater if the Ss had received more extended acquisition training. The interpretation of these findings is difficult because of the confounding of the reinforcement schedule with the level of responding that was in effect during the test phase of the experiment. It is obvious from inspection of Fig. 1 that the CRF Ss tended to respond more during the dummy intervals than did the VI animals $(U=462, p<.05)$. Thus, even though both groups were tested after meeting the same 3-min no-response criterion, this did not assure that the response rate in the absence of extraneous stimulation would be at the same low level. The difficult question concerns how to interpret the stimulus-induced inhibition of the CRF animals. Although it is true that the baseline response rate during the test session was higher for the CRF than for the VI Ss, it was still not much different from the two responses per minute that had led to a significant disinhibition effect in previous experiments (Brimer, 1970). But in all these previous experiments, animals had been trained initially with a partial reinforcement schedule. Perhaps animals originally trained under a CRF schedule will never disinhibit. Or perhaps CRF-trained Ss will show disinhibition only when their baseline response rate has been reduced to a level far below that which is necessary with VI-trained animals. Or perhaps CRF animals will display disinhibition only when extensive acquisition training is given prior to extinction. Experiment 2 attempted to clarify this situation by testing CRF-trained animals at different stages of extinction.

\section{EXPERIMENT 2 \\ Method}

The Ss were 60 male hooded rats, divided into six experimental groups and maintained at approximately $75 \%$ of their ad lib weights throughout the experiment. After initial magazine training, the Ss were given 50 trials a day leverpressing for food under a CRF schedule. Half the Ss received 100 reinforcements, and the other half, 500 reinforcements, prior to the introduction of extinction training and testing for disinhibition. For the different groups of Ss, the disinhibition test was introduced at different stages of extinction, as determined by no-response criteria of 6,9 , and $12 \mathrm{~min}$. As before, disinhibition was indexed by the difference in responding that occurred during the 3 -min stimulus and dummy intervals.

Results and Discussion

The results of the test for the different experimental groups are presented in Fig. 2. The relevant data for the $3-\mathrm{min}$ no-response groups of Experiment 1 are also included for comparison. It should be noted that in both the 100 and 500 reinforcement conditions, the dummy response rates progressively decreased as the extinction criteria was increased. As previously noted, the extraneous stimulus presented early in extinction produces a decrement in responding. However, as extinction training was continued, there came a point where the level of responding increased during the stimulus interval, even though the baseline level of responding continued to decrease. This occurred after 9 min with the $100 \mathrm{CRF}$ animals and after 12 min with the 500 CRF Ss. It is at just these two points that significant disinhibition can be demonstrated by comparing the stimulus and dummy response rates $(T=8.0, p<.05$, and $\mathrm{T}=3.0, \mathrm{p}<.01$, respectively). Thus, it seems clear that disinhibition may occur after original training on a CRF schedule if the extinction criterion is made sufficiently stringent.

After 100 continuous reinforcements and extinction training to a 12-min no-response criterion, significant disinhibition could not be demonstrated $(\mathrm{T}=21.0, \mathrm{p}>.05)$. This provides further evidence in support of an earlier suggestion (Brimer, 1970) that the response rate must be within some low critical range for the disinhibition phenomenon to occur. There is also some evidence in the present results that the critical level may be a function of the amount of initial reinforced training. For example, the dummy response rates in the 12-min group tested after 100 reinforcements were not different from the rates displayed by the comparable 500 animals ( $U=49.5, p>.05)$. However, only in the case of the latter Ss was the disinhibition effect in evidence. One might argue that comparisons of the baseline rates are not very convincing because the number of responses emitted during the dummy intervals were so low that it would have been difficult to show any reliable differences. However, as the no-response criterion was increased, the length of the extinction training session and the number of unreinforced responses emitted prior to testing increased accordingly. Thus, it seems reasonable to conclude that the different no-response groups were tested at progressively greater "depths" of extinction. This, in turn, suggests that the amount of extinction training that is necessary to demonstrate significant disinhibition increases with the amount of continuously reinforced training trials.

It is interesting to note that in previous studies employing a partial-reinforcement training schedule, the evidence suggested that disinhibition would not occur until the response rate was reduced to a level of 1 to 2 responses per minute (Experiment 1, this paper; Brimer, 1970). In marked contrast, the results of the present study suggest that a far lower level of responding (both mean and median dummy rates of less than 0.5 responses per minute) is necessary to show disinhibition after original training on a CRF schedule. In an earlier experiment (Brimer, 1970, Experiment 6), an attempt was made to test for disinhibition after a single reinforcement which produced a baseline response rate of approximately 2 to 3 responses per minute. The failure to obtain disinhibition at that stage of training was taken as evidence that a low response rate was not a sufficient condition for disinhibition. Rather, it was suggested that the response rate had to be reduced from some previously higher level. In view of the present findings, it seems clear that the failure to demonstrate disinhibition was inevitable. Consequently, the conclusion drawn from earlier studies should be modified to read: That for practical, if not necessarily for theoretical reasons, a low response rate is not a sufficient condition for the demonstration of the disinhibition phenomenon.

\section{REFERENCES}

BRIMER, C. J. Disinhibition of an operant response. Learning \& Motivation, 1970, in press. 